\title{
Editorial
}

\section{Uso y relevancia de las publicaciones odontológicas en español para estudiantes de pre y posgrado}

\author{
Use and relevance of dental \\ publications in Spanish \\ language for undergraduate and \\ postgraduate students
}

Ibraín Enrique Corrales-Reyes ${ }^{1, a}$

${ }^{1}$ Universidad de Ciencias Médicas de Granma, Hospital General Universitario Carlos Manuel de Céspedes, Bayamo, Cuba.

a Licenciado en Estomatología. Residente de II año de Cirugía Maxilofacial.

\section{Correspondencia:}

Ibraín Enrique Corrales-Reyes.

Correo electrónico: iecorralesr@infomed.sld.cu

Ignacio Pérez \# 12A e/ Avenida 26 de Julio y Donato Mármol. CP: 87300.

Jiguaní-Granma, Cuba. ORCID: 0000-0002-2146-9014.

Recibido: 05/07/19

Aceptado: 08/07/19

Publicado: 12/09/19
Durante algún tiempo, el ejercicio de la profesión odontológica se basó en el conocimiento clínico personal acumulado y el apego a los procedimientos históricamente sustentados, en lo que se podría denominar como "odontología basada en la experiencia" ${ }^{1}$. En la actualidad, el volumen de información que anualmente se publica en revistas científicas odontológicas es abrumador, por lo que, para mantenerse actualizado, es necesario leer diariamente un promedio de 19 artículos. El problema es que muchos de estos artículos no comunican resultados de investigaciones relevantes o adolecen de errores metodológicos. Entonces, el modelo clásico, que concibe al odontólogo como un almacenador de información, ha cambiado al considerarlo como un especialista manejador de información ${ }^{1}$.

Con el cambio de paradigma, la práctica odontológica pasó de estar basada en la experiencia a basada en la evidencia, surgiendo así la Odontología Basada en Evidencias (OBE), que aboga por el uso racional de la mejor evidencia disponible unida a la experiencia clínica del profesional para que, con el consentimiento del paciente, se recomiende y aplique el mejor tratamiento en el momento oportuno. De esta forma, se interrelacionan dos procesos, de un lado, la revisión sistemática de la evidencia disponible y, del otro, su introducción y evaluación en la terapéutica ${ }^{1}$.
La razón que justifica la necesidad de investigar y consumir información en las facultades de ciencias médicas, es la de preparar a los estudiantes con habilidades y conocimientos nuevos con una adecuada formación académica, en un mundo en acelerado desarrollo. Es en este escenario donde el estudiante de Odontología debe aprender a seleccionar la mejor evidencia científica disponible.

Una laguna del conocimiento, la necesidad de obtener notas satisfactorias o el contacto con una enfermedad odontológica que no conoce suficientemente constituyen motivaciones para que el propio estudiante diseñe estrategias para acceder a la información. Entonces surgen las siguientes interrogantes: ¿Qué evidencias científicas seleccionar y en qué idioma buscarlas? ¿Actualmente los estudiantes de pre y postgrado de Odontología buscan y leen información científica de revistas odontológicas en español o sólo consumen información en inglés? ¿Es útil y relevante para un estudiante de Odontología lo que se publica en las revistas odontológicas en español? ¿Cubre sus necesidades de pertinencia y actualización para los estudios que realiza?

Si bien es cierto que el inglés es el idioma de la ciencia, y que algunos países desarrollados en términos de ciencia, tecnología y economía, cuyo idioma oficial no es el 
inglés, plantean publicar sus revistas en este idioma, el español debe sobrevivir por disponer de un "mercado" común con los países latinoamericanos ${ }^{2}$. No se trata de negar el inglés como idioma oficial de la ciencia, sino de defender el español, de manera que coexista con otros idiomas.

El hecho de que un artículo científico se publique en inglés no es garantía de calidad. Por lo tanto, se requieren habilidad, experiencia y dedicación para la lectura crítica, de manera que se pueda discernir correctamente entre lo útil y lo prescindible en términos de ciencia. Para profesionales y estudiantes, queda claro que consumir la ciencia odontológica regional, generalmente publicada en español, es útil e inteligente por cuanto permite establecer comparaciones a este nivel, donde los problemas son comunes y las soluciones similares.
Tras una revisión de la colección SciELO regional, se puede constatar que existe más de una veintena de revistas odontológicas, con o sin vigencia de indexación actual, que publican constantemente o han publicado, investigaciones realizadas en los países latinoamericanos que perfectamente pueden ser utilizadas en la praxis de la OBE. Lo importante ahora es concientizar a los estudiantes y profesionales de la necesidad de consumir la ciencia que se genera y publica en español en las revistas odontológicas.

\section{Referencias bibliográficas}

1. Uribe S. ¿Qué es la odontología basada en la evidencia? Rev Fac Odontol Valparaiso. 2000;2(4):281-287.

2. González de Dios J, González C. El español como vehículo de transmisión de los avances científicos. Emergencias. 2014;26:406-410. 